\title{
A product property of Sobolev spaces with application to elliptic estimates
}

\author{
Henry C. Simpson (*) - Scott J. Spector (**)
}

ABSTRACT - In this paper a Sobolev inequality, which generalizes the ordinary Banach algebra property of such spaces, is established; for $p \in[1, \infty), n, m \in Z^{+}$, and $m \geq 2$ that satisfy $m>n / p$,

$$
\|\phi \psi\|_{m, p, \Omega} \leq K\left[\left(\sup _{\Omega_{s}}|\phi|\right)\|\psi\|_{m, p, \Omega}+\left(\|\psi\|_{m-1, q, \Omega}+\|\psi\|_{m-1, p, \Omega}\right)\|\phi\|_{m, p, \Omega}\right]
$$

for all $\phi, \psi \in W^{m, p}(\Omega)$ that satisfy spt $\psi \subset \Omega_{s} \subset \Omega$ and domains $\Omega \subset \mathbb{R}^{n}$ that are nonempty, open, and satisfy the cone condition. Here $q=p$ if $p>n$, $q \in(n / \Upsilon, p n /(n-p)]$ if $n>p, q \in(n / \Upsilon, \infty)$ if $p=n, K=K(n, p, m, q, \mathcal{C})$, where $\mathcal{C}$ is the cone from the cone condition, and $\Upsilon:=\llbracket n / p \rrbracket$, the largest integer less than or equal to $n / p$.

Mathematics Subject Classification (2010). 46E35, 35J57, 74B15.

KEYwORDS. Elasticity, elliptic regularity, Sobolev estimate, systems of partial differential equations.

\section{Introduction; Sobolev Spaces}

A standard classical methodology used to obtain a priori estimates for elliptic systems of partial differential equations is to first prove the required estimate when the system has constant coefficients and the region has smooth boundary and then use a partition of unity to extend the es-

(*) Indirizzo dell'A.: Department of Mathematics, University of Tennessee, Knoxville, TN 37996-1300, USA.

E-mail: hsimpson@math.utk.edu

(**) Indirizzo dell'A.: Department of Mathematics, Southern Illinois University, Carbondale, IL 62901, USA.

E-mail: sspector@siu.edu 
timate to coefficients that depend on position and regions that are less regular. For example, Agmon, Douglis, and Nirenberg [3, 4] first establish the estimate (in the notation from Elasticity): for all $\boldsymbol{u} \in C^{\infty}\left(\bar{\Omega} ; \mathbb{R}^{n}\right)$ that satisfy $\boldsymbol{u}=\mathbf{0}$ in a neighborhood of $\mathcal{D}:=\partial \Omega \backslash \mathcal{S}$,

$$
\|\boldsymbol{u}\|_{m+1, p, \Omega} \leq N\left[\|\operatorname{Div} \mathrm{C}[\nabla \boldsymbol{u}]\|_{m-1, p, \Omega}+\|\mathrm{C}[\nabla \boldsymbol{u}] \boldsymbol{n}\|_{m-\frac{1}{p}, p, \mathcal{S}}+\|\boldsymbol{u}\|_{p, \Omega}\right],
$$

where $\mathrm{C}: \mathrm{M}^{n \times n} \rightarrow \mathrm{M}^{n \times n}$ is a constant linear mapping of the $n \times n$ matrices $\mathrm{M}^{n \times n}$ and $\Omega$ is a ball with $\mathcal{S}=\varnothing$ or $\Omega$ is a half-ball and $\mathcal{S}$ is the flat portion of the boundary of $\Omega$. Here $m \in Z^{+}, p \in(1, \infty), \boldsymbol{n}$ is the outward unit normal to the boundary $\partial \Omega$,

$$
\begin{aligned}
\|\boldsymbol{u}\|_{p, \Omega}^{p}: & :=\int_{\Omega}|\boldsymbol{u}(\boldsymbol{x})|^{p} \mathrm{~d} \boldsymbol{x}, & (\nabla \boldsymbol{u})_{i} & :=\frac{\partial \boldsymbol{u}}{\partial x_{i}}, \\
\|\boldsymbol{u}\|_{m, p, \Omega}^{p} & :=\sum_{|\boldsymbol{a}| \leq m}\left\|D^{a} \boldsymbol{u}\right\|_{p, \Omega}^{p}, & (\operatorname{Div} M)_{i} & :=\sum_{j=1}^{n} \frac{\partial M_{i j}}{\partial x_{j}},
\end{aligned}
$$

and $\boldsymbol{a}=\left(\alpha_{1}, \ldots, \alpha_{n}\right)$ is a multi-index with $|\boldsymbol{a}|=\alpha_{1}+\cdots+\alpha_{n}$ and $D^{\alpha}=\partial_{x_{1}}^{\alpha_{1}} \ldots \partial_{x_{n}}^{\alpha_{n}}$.

For a general bounded open region $\Omega \subset \mathbb{R}^{n}$, a suitable open covering of $\Omega$ and $\partial \Omega$, respectively, by balls and half-balls, together with a partition of unity can then be used (see [3]) to prove (1.1) for $\mathrm{C}(\boldsymbol{x}): \mathrm{M}^{n \times n} \rightarrow \mathrm{M}^{n \times n}$ whose components are $m$-times continuously differentiable on $\bar{\Omega}$. More generally, if one wants to establish ${ }^{1}(1.1)$ for $\mathrm{C} \in W^{m, p}(\Omega)$, then one can make use of Moser's [7, pp. 273-274] tame inequality (see Klainerman and Majda [6, pp. 516-517] for a nice proof): If $1 \leq p<\infty$ and $k \in Z^{+}$, then there exists a constant $C=C(n, p, k)>0$ such that

$$
C^{-1}\|\phi \psi\|_{k, p, \mathbb{R}^{n}} \leq\|\phi\|_{\infty, \mathbb{R}^{n}}\|\psi\|_{k, p, \mathbb{R}^{n}}+\|\psi\|_{\infty, \mathbb{R}^{n}}\|\phi\|_{k, p, \mathbb{R}^{n}}
$$

for all $\phi, \psi \in W^{k, p}\left(\mathbb{R}^{n}\right) \cap L^{\infty}\left(\mathbb{R}^{n}\right)$.

However, (1.2) is an inequality for Sobolev functions defined on all of $\mathbb{R}^{n}$, while in practice one must make use of this inequality for Sobolev functions defined on a bounded open region $\Omega \subset \mathbb{R}^{n}$. This presents no difficulties when the boundary of $\Omega$ is sufficiently smooth since one can

( $\left.{ }^{1}\right)$ See, e.g., [12] for a proof of (1.1) when C is a Sobolev function. See, e.g., Ragusa [11] and the references therein for interior regularity when $C$ is discontinuous. 
then use standard extension results to obtain a version of (1.2) for such domains. When the boundary of the region is not smooth there are some unresolved difficulties. ${ }^{2}$

The main purpose of this paper is to provide a partial resolution of these difficulties by proving an inequality, which is similar to (1.2) and which is also useful for elliptic estimates, for regions that satisfy (only) a cone condition. In particular we show that if $\Omega \subset \mathbb{R}^{n}$ is nonempty, open, and satisfies the cone condition and if $p \in[1, \infty), n, m \in Z^{+}, m \geq 2$, and $p \in(n / m, n)$, then for any $q \in\left(n / \llbracket \frac{n}{p} \rrbracket, p n /(n-p)\right]$ there is a constant $K=K(n, p, m, q, \mathcal{C})$ such that

$$
\begin{aligned}
& \left\|\phi_{1} \phi_{2}\right\|_{m, p, \Omega} \leq \\
& \quad K\left[\left(\sup _{\Omega_{s}}\left|\phi_{1}\right|\right)\left\|\phi_{2}\right\|_{m, p, \Omega}+\left(\left\|\phi_{2}\right\|_{m-1, q, \Omega}+\left\|\phi_{2}\right\|_{m-1, p, \Omega}\right)\left\|\phi_{1}\right\|_{m, p, \Omega}\right]
\end{aligned}
$$

for all $\phi_{1}, \phi_{2} \in W^{m, p}(\Omega)$ that satisfy spt $\phi_{2} \subset \Omega_{s} \subset \Omega$. Here $\mathcal{C}$ is the cone from the cone condition ${ }^{3}$ and $\llbracket x \rrbracket$ is the largest integer less than or equal to $x$.

We note that our inequality, unlike (1.2), has the interesting feature that its dependence on the supremum of the first function is limited to the region that supports the second function. Our initial motivation for studying such inequalities originated in problems of global bifurcation ${ }^{4}$ for the stronglyelliptic system that governs the equilibrium of elastic materials. In this context inequality (1.3) extends results of Valent [13, pp. 22-27] that are used to improve estimates in [3, 4] in order to apply them to elasticity. Our proof makes use of the following special cases of the usual Sobolev inequalities.

Proposition (See, e.g., [2, pp. 85-86]). Let $\Omega \subset \mathbb{R}^{n}, n \geq 1$, be a nonempty open region that satisfies the cone condition. Suppose $1 \leq p<\infty$, $k \in Z^{+}$, and $j \in \mathbb{N}$. Define $p_{k}:=p n /(n-k p)$ if $n>k p$ and $p_{k}:=\infty$ otherwise. Then there exists a constant $K=K(n, p, k, j, q, \mathcal{C})$, where $\mathcal{C}$ is the cone from the cone condition, that has the following properties.

( $\left.{ }^{2}\right)$ See Maz'ya and Shaposhnikova [8, Chapter 7] for regions whose boundary can be parametrized by an appropriate Sobolev function. See, also, Nečas [10].

$\left({ }^{3}\right)$ That is, every $\boldsymbol{x} \in \Omega$ is the vertex of cone that is contained in $\Omega$ and is a rigid deformation of $\mathcal{C}$. See, e.g., [1, p. 66] or [2, p. 82].

$\left({ }^{4}\right)$ See, e.g., $[5,9]$. 
I. (Sobolev Imbedding Theorem) If $k>n / p$ then $W^{k, p}(\Omega) \subset C_{B}(\Omega)$ with

$$
\sup _{\Omega}|\phi| \leq K\|\phi\|_{k, p, \Omega} \text { for all } \phi \in W^{k, p}(\Omega) .
$$

II. (Gagliardo-Nirenberg-Sobolev Inequality) If $k \leq n / p$ then $W^{k+j, p}(\Omega) \subset$ $W^{j, q}(\Omega)$ with

$$
\|\phi\|_{j, q, \Omega} \leq K\|\phi\|_{k+j, p, \Omega} \text { for all } \phi \in W^{k+j, p}(\Omega)
$$

and $q \in\left[p, p_{k}\right]$ if $p_{k}<\infty$ and $q \in[p, \infty)$ otherwise.

Here,

$$
C_{B}(\Omega):=\left\{\phi \in C(\Omega): \phi \in L^{\infty}(\Omega)\right\},
$$

which is a Banach space under the $L^{\infty}$-norm.

\section{The Product Property}

For a Sobolev function $\psi \in W^{k, p}(\Omega), k \in \mathbb{N}, p \in[1, \infty)$, we define the support of $\psi$ by

spt $\psi:=\Omega \backslash\{\boldsymbol{x} \in \Omega: \psi(\boldsymbol{z})=0$ for a.e. $\boldsymbol{z}$ in some open neighborhood of $\boldsymbol{x}\}$.

Thus, since the complement of spt $\psi$ is an open set, $D^{\alpha} \psi=0$ a.e. on $\Omega \backslash(\operatorname{spt} \psi)$ for $|\alpha| \leq k$. Consequently, if $\phi, \psi \in W^{k, p}(\Omega), k>n / p$, and $\operatorname{spt} \psi \subset \Omega_{s}$ then $\phi\left(D^{\gamma} \psi\right) \in L^{p}(\Omega)$ for $|\gamma| \leq k$ and

$$
\left\|\phi\left(D^{\gamma} \psi\right)\right\|_{p, \Omega} \leq\left(\sup _{\Omega_{s}}|\phi|\right)\left\|D^{\gamma} \psi\right\|_{p, \Omega} .
$$

The main result of this paper is the following theorem, which generalizes the usual Banach algebra property of $W^{m, p}, m>n / p$. See Valent [13, pp. 22-27] for similar results.

THEOREM. Let $\Omega \subset \mathbb{R}^{n}$, $n \in Z^{+}$, be a nonempty open region that satisfies the cone condition. Suppose $1 \leq p<\infty, m \in Z^{+}$with $m>n / p$, and $\Omega_{s} \subset \Omega$ is measurable. Then for every $q \in\left(\frac{n}{\Upsilon}, n p /(n-p)\right]$, if $n>p$, and for every $q \in\left(\frac{n}{\Upsilon}, \infty\right)$, if $p=n$, there exists a constant $K=$ $K(n, p, m, q, \mathcal{C})>0$, where $\mathcal{C}$ is the cone from the cone condition for $\Omega$ and $\Upsilon:=\llbracket \frac{n}{p} \rrbracket$, such that the following are satisfied. 
(a) If $m=1$ then for all $\phi, \psi \in W^{1, p}(\Omega)$ that satisfy $\operatorname{spt} \psi \subset \Omega_{s} \subset \Omega$

$$
\|\phi \psi\|_{1, p, \Omega} \leq 2\left[\left(\sup _{\Omega_{s}}|\phi|\right)\|\psi\|_{1, p, \Omega}+\left(\sup _{\Omega}|\psi|\right)\|\phi\|_{1, p, \Omega}\right] .
$$

(b) If $m \geq 2$ and $n \geq p$ then for all $\phi, \psi \in W^{m, p}(\Omega)$ that satisfy spt $\psi \subset \Omega_{s} \subset \Omega$

$$
\|\phi \psi\|_{m, p, \Omega} \leq K\left[\left(\sup _{\Omega_{s}}|\phi|\right)\|\psi\|_{m, p, \Omega}+\left(\|\psi\|_{m-1, q, \Omega}+\|\psi\|_{m-1, p, \Omega}\right)\|\phi\|_{m, p, \Omega}\right] .
$$

(c) If $m \geq 2$ and $p>n$ then for all $\phi, \psi \in W^{m, p}(\Omega)$ that satisfy $\operatorname{spt} \psi \subset \Omega_{s} \subset \Omega$

$$
\|\phi \psi\|_{m, p, \Omega} \leq K\left[\left(\sup _{\Omega_{s}}|\phi|\right)\|\psi\|_{m, p, \Omega}+\|\psi\|_{m-1, p, \Omega}\|\phi\|_{m, p, \Omega}\right] .
$$

REMARK. Note that the constant $K$ is independent of $\Omega_{s}$. When $\Omega$ has finite volume one can combine the term $\|\psi\|_{m-1, p, \Omega}$ with its upper bound $\|\psi\|_{m-1, q, \Omega}$ in (2.3), however, the constant $K$ will then depend on the volume of $\Omega$.

Proof of (2.2). To simplify the notation we drop the $\Omega$, but leave the $\Omega_{s}$, on the appropriate norms. To prove (2.2) we first note that, since $p>n$, without loss of generality we may assume, by the Sobolev imbedding theorem, that $\phi, \psi \in W^{1, p}(\Omega) \cap C_{B}(\Omega)$. Next, $\|\phi \psi\|_{1, p} \leq\|\phi \psi\|_{0, p}+\|\nabla(\phi \psi)\|_{0, p}$ and, in view of (2.1),

$$
\|\phi \psi\|_{0, p} \leq\left(\sup _{\Omega_{s}}|\phi|\right)\|\psi\|_{1, p}
$$

However, $\nabla(\phi \psi)=\phi \nabla \psi+\psi \nabla \phi$ so that, with the aid of (2.1),

$$
\begin{aligned}
\|\nabla(\phi \psi)\|_{0, p} & \leq\|\phi \nabla \psi\|_{0, p}+\|\psi \nabla \phi\|_{0, p} \\
& \leq\left(\sup _{\Omega_{s}}|\phi|\right)\|\psi\|_{1, p}+\left(\sup _{\Omega}|\psi|\right)\|\phi\|_{1, p},
\end{aligned}
$$

which proves (2.2).

Proof of (2.3) when $\llbracket \frac{n}{p} \rrbracket=m-1$ and $m \geq 2$. Note that $n \geq p$. Let $q \in\left(\frac{n}{m-1}, \frac{n p}{n-p}\right]$ if $n>p$ and $q \in\left(\frac{n}{m-1}, \infty\right)$ if $n=p$. To prove (2.3) when 
$\llbracket \frac{n}{p} \rrbracket=m-1$ we first note for future reference that

$$
q>p, \quad q(m-1)>n,
$$

and if $m>2$ then $n>p$ and

$$
\frac{n p}{n-p} \leq \frac{n}{m-2}
$$

Now, let $\phi, \psi \in W^{m, p}(\Omega)$. Then, since $m p>n, \phi, \psi \in C_{B}(\Omega)$ by the Sobolev imbedding theorem, while $q \in\left[p, p_{1}\right]$ (or $q \in[p, \infty)$ ) yields $\psi \in W^{m-1, q}(\Omega)$ by the Gagliardo-Nirenberg-Sobolev inequality. Next,

$$
\begin{aligned}
\|\phi \psi\|_{m, p} & \leq \sum_{|\alpha| \leq m}\left\|D^{\alpha}(\phi \psi)\right\|_{0, p} \\
& =\sum_{|\alpha| \leq m}\left\|\sum_{|\beta|+|\gamma|=|\alpha|} c_{\beta \gamma}\left(D^{\beta} \phi\right)\left(D^{\gamma} \psi\right)\right\|_{0, p} \\
& \leq K \sum_{|\alpha| \leq m} \sum_{|\beta|+|\gamma|=|\alpha|}\left\|\left(D^{\beta} \phi\right)\left(D^{\gamma} \psi\right)\right\|_{0, p},
\end{aligned}
$$

where $K:=\max c_{\beta \gamma}$ only depends on $n$ and $m$.

If $|\beta|=0$ then $|\gamma| \leq m$ and hence by (2.1)

$$
\left\|\phi\left(D^{\gamma} \psi\right)\right\|_{0, p} \leq\|\phi\|_{\infty, \Omega_{s}}\left\|D^{\gamma} \psi\right\|_{0, p} \leq\|\phi\|_{\infty, \Omega_{s}}\|\psi\|_{m, p} .
$$

If $|\beta|=m$ then $|\gamma|=0$ and hence by the Sobolev imbedding theorem and $(2.5)_{2}$

$$
\left\|\left(D^{\beta} \phi\right) \psi\right\|_{0, p} \leq\left\|D^{\beta} \phi\right\|_{0, p}\|\psi\|_{\infty} \leq K\|\phi\|_{m, p}\|\psi\|_{m-1, q} .
$$

If $|\gamma|=m-1$ (and $|\beta| \neq 0$ ) then $|\beta|=1$. Define $q^{\prime}$ so that $\frac{1}{q}+\frac{1}{q^{\prime}}=\frac{1}{p}$ and $p_{m-1}:=p n /(n-(m-1) p)$ if $n>(m-1) p$ and $p_{m-1}:=\infty$ otherwise. Then, by $(2.5)_{2}, q^{\prime} \in\left(p, p_{m-1}\right)$ and hence by Hölder's inequality and the Gagliardo-Nirenberg-Sobolev inequality $(k=m-1)$

$$
\begin{gathered}
\left\|\left(D^{\beta} \phi\right)\left(D^{\gamma} \psi\right)\right\|_{0, p} \leq\left\|D^{\beta} \phi\right\|_{0, q^{\prime}}\left\|D^{\gamma} \psi\right\|_{0, q} \leq \\
\|\phi\|_{1, q^{\prime}}\|\psi\|_{m-1, q} \leq K\|\phi\|_{m, p}\|\psi\|_{m-1, q} .
\end{gathered}
$$

Finally, if $2 \leq|\beta| \leq m-1$ then $|\gamma| \leq m-2$. Note $|\beta|+|\gamma| \leq m, m \geq 3$, and $n \neq p$ (since $n / p \geq m-1 \geq 2$ ). Thus, by Hölder's inequality,

$$
\begin{aligned}
\left\|\left(D^{\beta} \phi\right)\left(D^{\gamma} \psi\right)\right\|_{0, p} & \leq\left\|D^{\beta} \phi\right\|_{0, \frac{p m}{n-(m-|\beta|) p}}\left\|D^{\gamma} \psi\right\|_{0, \frac{p n}{(m-\beta) p}} \\
& \leq\|\phi\|_{|\beta|, \frac{p m}{n-(m-\beta \mid) p}}\|\psi\|_{m-|\beta|, \frac{p m}{(m-|\beta|) p}} .
\end{aligned}
$$


Then, in view of the Gagliardo-Nirenberg-Sobolev inequality $(k=m-|\beta|$ and $k=|\beta|-1)$,

$$
\|\phi\|_{|\beta|, \frac{p n}{n-(m-\mid \beta) p}} \leq K\|\phi\|_{m, p}, \quad\|\psi\|_{m-|\beta|, \frac{n}{m-|\beta|}} \leq K\|\psi\|_{m-1, q},
$$

since $q \in\left(\frac{n}{m-1}, \frac{n}{m-2}\right]$ by (2.6) and the definition of $q$. The desired result, (2.3), now follows in the case when $\llbracket \frac{n}{p} \rrbracket=m-1$ from (2.7) $-(2.12)$.

Proof of (2.3) when $1 \leq \llbracket \frac{n}{p} \rrbracket<m-1$. We prove (2.3) by induction on $m$. Note that $n \geq p$. Define $\widehat{m}:=\Upsilon+1=\llbracket \frac{n}{p} \rrbracket+1$. Then $\widehat{m}>n / p \geq$ $\widehat{m}-1$ and $\widehat{m} \geq 2$. The induction starts at $m=\widehat{m}$. Then, as we have just proven, (2.3) is valid for $m=\widehat{m}$ and any $q$ in the appropriate interval. To continue the induction we assume (2.3) is valid, for some $m \geq \widehat{m}$ and $q$, and show it is valid for $m+1$ and the same $q$.

Let $\phi, \psi \in W^{m+1, p}(\Omega)$. Then $q \in\left(\frac{n}{\Upsilon}, \frac{n p}{n-p}\right]$ if $n>p$ and $q \in\left(\frac{n}{\Upsilon}, \infty\right)$ if $n=p$; consequently $\psi \in W^{m, q}(\Omega)$ by the Gagliardo-Nirenberg-Sobolev inequality. We again note $\nabla(\phi \psi)=\psi \nabla \phi+\phi \nabla \psi$ and hence

$$
\begin{aligned}
\|\phi \psi\|_{m+1, p} & \leq\|\phi \psi\|_{m, p}+\|\nabla(\phi \psi)\|_{m, p} \\
& \leq\|\phi \psi\|_{m, p}+\|\psi \nabla \phi\|_{m, p}+\|\phi \nabla \psi\|_{m, p} .
\end{aligned}
$$

However, by the induction hypothesis

$$
\begin{aligned}
\|\phi \psi\|_{m, p} & \leq K\left[\left(\sup _{\Omega_{s}}|\phi|\right)\|\psi\|_{m, p}+\left(\|\psi\|_{m-1, q}+\|\psi\|_{m-1, p}\right)\|\phi\|_{m, p}\right] \\
& \leq K\left[\left(\sup _{\Omega_{s}}|\phi|\right)\|\psi\|_{m+1, p}+\left(\|\psi\|_{m, q}+\|\psi\|_{m, p}\right)\|\phi\|_{m+1, p}\right],
\end{aligned}
$$

and, similarly,

$$
\begin{gathered}
\|\phi \nabla \psi\|_{m, p} \leq K\left[\left(\sup _{\Omega_{s}}|\phi|\right)\|\psi\|_{m+1, p}+\left(\|\psi\|_{m, q}+\|\psi\|_{m, p}\right)\|\phi\|_{m+1, p}\right] \\
\|\psi \nabla \phi\|_{m, p} \leq K\left[\left(\sup _{\Omega}|\nabla \phi|\right)\|\psi\|_{m, p}+\left(\|\psi\|_{m, q}+\|\psi\|_{m, p}\right)\|\phi\|_{m+1, p}\right]
\end{gathered}
$$

since $\Omega_{s} \subset \Omega$, while by the Sobolev imbedding theorem $(k=m)$

$$
\sup _{\Omega}|\nabla \phi| \leq K\|\phi\|_{m+1, p}
$$


Inequality (2.3) with $m$ replaced by $m+1$, now follows from (2.13)(2.17).

Proof of (2.4). Note that $p>n$. First we prove (2.4) for $m=2$. Let $\phi, \psi \in W^{2, p}(\Omega)$. Then (2.13) with $m=1$ is valid, however, (2.14)-(2.16) must be replaced by (see (2.2))

$$
\begin{gathered}
\|\phi \psi\|_{1, p} \leq 2\left[\left(\sup _{\Omega_{s}}|\phi|\right)\|\psi\|_{1, p}+\left(\sup _{\Omega}|\psi|\right)\|\phi\|_{1, p}\right], \\
\|\psi \nabla \phi\|_{1, p} \leq 2\left[\left(\sup _{\Omega}|\nabla \phi|\right)\|\psi\|_{1, p}+\left(\sup _{\Omega}|\psi|\right)\|\nabla \phi\|_{1, p}\right],
\end{gathered}
$$

and an appropriate estimate for $\|\phi \nabla \psi\|_{1, p}$. Clearly, $\|\cdot\|_{1, p} \leq\|\cdot\|_{2, p}$ and since $p>n$ the Sobolev imbedding theorem $(k=1)$ yields

$$
\sup _{\Omega}|\psi| \leq K\|\psi\|_{1, p}, \quad \sup _{\Omega}|\nabla \phi| \leq K\|\nabla \phi\|_{1, p} \leq K\|\phi\|_{2, p} .
$$

Thus, we need only estimate the term $\|\phi \nabla \psi\|_{1, p}$, which replaces (2.15), to finish the proof when $m=2$.

We note $\nabla(\phi \nabla \psi)=\phi \nabla \nabla \psi+\nabla \psi \otimes \nabla \phi$ and hence

$$
\begin{aligned}
\|\phi \nabla \psi\|_{1, p} & \leq\|\phi \nabla \psi\|_{0, p}+\|\nabla(\phi \nabla \psi)\|_{0, p} \\
& \leq\|\phi \nabla \psi\|_{0, p}+\|\phi \nabla \nabla \psi\|_{0, p}+\|\nabla \psi \otimes \nabla \phi\|_{0, p} .
\end{aligned}
$$

In view of (2.1) the first two terms on the right-hand side of (2.20) satisfy

$$
\|\phi \nabla \psi\|_{0, p} \leq\left(\sup _{\Omega_{s}}|\phi|\right)\|\psi\|_{2, p}, \quad\|\phi \nabla \nabla \psi\|_{0, p} \leq\left(\sup _{\Omega_{s}}|\phi|\right)\|\psi\|_{2, p}
$$

while the last term on the right-hand side of (2.20) satisfies

$$
\|\nabla \psi \otimes \nabla \phi\|_{0, p} \leq\left(\sup _{\Omega}|\nabla \phi|\right)\|\psi\|_{1, p} .
$$

Inequality (2.4) with $m=2$ now follows from (2.13) with $m=1$ and (2.18)-(2.22).

To complete the proof we note that (2.4) can be obtained by induction on $m$ for $m>2$. However, the required steps are identical to those in the proof of (2.3), when $1 \leq \llbracket \frac{n}{p} \rrbracket<m-1$, with the terms $\|\psi\|_{m, q}$ and $\|\psi\|_{m-1, q}$ deleted. 
REMARK. If $n \geq p \geq 1, m \geq \widehat{m}=\Upsilon+1:=\llbracket \frac{n}{p} \rrbracket+1 \geq 2$, and $\Omega_{s} \subset \Omega$ is open then one can show that, for all $\phi, \psi \in W^{m, p}(\Omega)$ that satisfy spt $\psi \subset \Omega_{s} \subset \Omega$ and for every $q \in\left(\frac{n}{r}, n p /(n-p)\right]$, if $n>p$, and for every $q \in\left(\frac{n}{\Upsilon}, \infty\right)$, if $p=n$, there exists a constant $K=K(n, p, m, q, \mathcal{C})>0$ such that

$$
\|\phi \psi\|_{m, p, \Omega} \leq K\left(\sum_{k=0}^{m-\widehat{m}}\left[\|\phi\|_{C_{B}^{k}\left(\Omega_{s}\right)}\|\psi\|_{m-k, p, \Omega}\right]+\|\psi\|_{m-1, q, \Omega}\|\phi\|_{m, p, \Omega}\right),
$$

where

$$
\|\phi\|_{C_{B}^{k}\left(\Omega_{s}\right)}:=\sum_{|\alpha| \leq k} \sup _{\boldsymbol{x} \in \Omega_{s}}\left|D^{\alpha} \phi(\boldsymbol{x})\right| .
$$

Inequality (2.23) is obtained by induction on $m$. The initial step is the above proof of (2.3) when $\llbracket \frac{n}{p} \rrbracket=m-1$. The induction is then similar to that presented above in the proof of (2.3) when $1 \leq \llbracket \frac{n}{p} \rrbracket<m-1$. The only significant difference is that one does not use the estimate (2.17), but instead leaves the appropriate version of (2.16) as it is, since each step in the induction argument will now add an additional derivative to the $\phi$ term that multiplies $\|\psi\|_{m-k, p}$.

Acknowledgments. The work of SJS was partially supported by the National Science Foundation under Grant No. DMS-1107899.

\section{REFERENCES}

[1] R. A. Adams, Sobolev Spaces, Academic Press, 1975.

[2] R. A. Adams and J. J. F. Fournier, Sobolev Spaces, $2^{\text {nd }}$ edition, Academic Press, 2003.

[3] S. Agmon, A. Douglis and L. Nirenberg, Estimates near the boundary for solutions of elliptic partial differential equations satisfying general boundary conditions. I, Comm. Pure Appl. Math. 12 (1959), 623-727.

[4] S. Agmon, A. Douglis and L. NiREnBerg, Estimates near the boundary for solutions of elliptic partial differential equations satisfying general boundary conditions. II, Comm. Pure Appl. Math. 17 (1964), 35-92.

[5] T. J. Healey and H. C. Simpson, Global continuation in nonlinear elasticity, Arch. Rational Mech. Anal. 143 (1998), 1-28.

[6] S. KLAInerman and A. MAJDA, Singular limits of quasilinear hyperbolic systems with large parameters and the incompressible limit of compressible fluids, Comm. Pure Appl. Math. 34 (1981), 481-524.

[7] J. Moser, A rapidly convergent iteration method and non-linear partial differential equations. I, Ann. Scuola Norm. Sup. Pisa (3) 20 (1966), 265-315. 
[8] V. G. MaZ'Ya and T. O. Shaposhnikova, Theory of Multipliers in Spaces of Differentiable Functions, Pitman, 1985.

[9] J. SHI and X. WANG, On global bifurcation for quasilinear elliptic systems on bounded domains. J. Differential Equations, 246 (2009), 2788-2812.

[10] J. NEČAs, Les Méthodes Directes en Théorie des Équations Elliptiques, Masson et Cie, Éditeurs, Paris 1967.

[11] M. Ragusa, Continuity of the derivatives of solutions related to elliptic systems, Proc. Royal Soc. Edinburgh 136A (2006), 1027-1039.

[12] H. C. Simpson and S. J. Spector, Applications of estimates near the boundary to regularity of solutions in linearized elasticity, SIAM J. Math. Anal. 41 (2009), 923-935.

[13] T. Valent, Boundary Value Problems of Finite Elasticity, Springer, 1988.

Manoscritto pervenuto in redazione il 23 Luglio 2012. 